\title{
Processing speed and mental retardation: Deadline procedures indicate fixed and adjustable limitations
}

\author{
NEIL BREWER \\ National Police Research Unit, Adelaide, South Australia, Australia \\ and \\ GLEN A. SMITH \\ University of Melbourne, Parkville, Victoria, Australia
}

\begin{abstract}
Brewer and Smith (1984) showed that control mechanisms mediating speed-accuracy regulation contribute to retarded-nonretarded differences in processing speed, with poorly controlled trial-to-trial RT adjustments underlying the greater RT variability of retarded individuals. In Experiment 1, response deadlines controlled processing time, thus minimizing the influence of such control mechanisms. The obtained speed-accuracy relations showed that retarded subjects were unable to match nonretarded subjects' accuracy when responding as rapidly, thus indicating structural limitations on processing speed. The results of Experiment 2 showed, however, that significant adjustments to retarded subjects' processing speed-exceeding those produced by practice-are achievable. Extended training at a short deadline led to tighter control of RT adjustments, with substantial improvements in mean RT when subjects transferred to a self-paced RT task.
\end{abstract}

A major theoretical concern in studies of information processing and mental retardation has been the extent to which the performance of retarded individuals reflects fixed (i.e., structural) or adjustable (i.e., control process) processing limitations. This distinction has been particularly influential in studies of learning and memory (Borkowski \& Cavanaugh, 1979; Campione \& Brown, 1977; Detterman, 1979; Fisher \& Zeaman, 1973), in which measures of processing accuracy have generally been the dependent variables of interest. In this study, we are concerned with the nature of the limitations on speed of processing in retarded individuals.

Mildly retarded individuals generally can respond accurately on a wide variety of tasks involving relatively simple perceptual judgments and motor responses. Typically, however, their performance is characterized by slowness and considerable intraindividual variability in speed of responding when compared with that of individuals of average intelligence (Baumeister \& Kellas, 1968; Nettelbeck \& Brewer, 1981). A wide range of experimental paradigms for examining speed of information processing has been used by researchers in an attempt to trace the source of these performance characteristics to cognitive processes assumed to mediate rapid informa-

Financial support was provided by Australian Research Grants Scheme A28015379. We thank S. Vukmirovic, C. Phillips, and A. Shinkfield for assistance with data collection and processing; the staff and employees of NADRASCA Training Centre and VATMI Industries; and the reviewers for their thoughtful comments. Requests for reprints may be sent to Neil Brewer, NPRU, P.O. Box 370, Marden, SA 5070, Australia. tion processing. Taken together, this work has implicated inefficiencies at virtually all stages of information processing, including sensory storage, perceptual encoding, memory comparison, response selection, and programming (for reviews, see Brewer \& Smith, 1982; Maisto \& Baumeister, 1984; Nettelbeck \& Brewer, 1981).

Because the knowledge and strategic requirements associated with performance on many of the rapidprocessing tasks that have been typically used appear to be minimal, an implicit assumption in most of this work has been that the slower processing of retarded individuals reflects structural, or fixed, limitations. However, Brewer and Smith (1982) have reviewed evidence that suggests that control mechanisms mediating efficient information processing may also contribute to the slower performance of retarded individuals. Our subsequent work on processing speed and mental retardation (and other developmental aspects of speed of processing) has focused on the contribution of control mechanisms mediating speed and accuracy across all information-processing operations (Brewer, 1987; Brewer \& Smith, 1984, 1989).

Specifically, Brewer and Smith have argued that the efficient operation of control mechanisms that mediate adaptive trial-to-trial adjustments in speed of responding is central to the attainment and maintenance of fast, accurate performance on rapid-processing tasks. In rapidprocessing tasks, subjects typically trade off speed and accuracy, with slow and fast responding associated with high and low accuracy, respectively. Also, at long response times, large variations in speed may minimally affect accuracy, whereas small increases in speed at rela- 
tively short response times may produce a marked decline in accuracy (Pachella, 1974). Consequently, if subjects are to avoid responding overly quickly, and hence inaccurately, or more slowly than is necessary to sustain accurate performance, they must be able to monitor accuracy of responses and to control adjustments in speed of responding from trial to trial within quite narrow limits (Brewer \& Smith, 1982, 1984; Rabbitt, 1979, 1981).

Within this framework and using a self-paced, serial choice reaction time (RT) task, Brewer and Smith (1984) investigated whether the way that retarded individuals monitor and control processing speed and accuracy contributes to their typically slower performance. Examination of speed-accuracy tradeoff functions (generated by post hoc RT partitioning) and trial-to-trial transitions in speed and accuracy indicated that a major factor underlying the slower performance of retarded individuals was that their trial-to-trial RT adjustments were much less precise than were those made by nonretarded subjects. This lack of precision resulted in frequent undershooting and overshooting of the RT bands that should have been targeted on a given trial or sequence of trials. The net effect of these poorly controlled trial-to-trial RT adjustments was an inability to generate stable performance within relatively narrow, fast RT bands just safely above overly fast error RT levels.

As Brewer and Smith (1984) have pointed out, most rapid-processing tasks used to probe retarded-nonretarded differences are self-paced. As such, they require subjects to make ongoing, adaptive, and precise RT adjustments in order to maximize speed of responding while maintaining a low overall error rate. Overly coarse RT adjustments from trial to trial on the part of retarded subjects will, therefore, inevitably be reflected in greater variability and longer average response times than would be seen in nonretarded subjects operating at the same overall level of accuracy.

This suggests some interesting questions. For example, what would happen if the influence of this less precise control of RT adjustments was constrained by equating processing time for retarded and nonretarded subjects? If increments in accuracy associated with increasing processing time followed a similar pattern for both retarded and nonretarded individuals, the control mechanisms mediating speed-accuracy regulation (rather than structural differences in the capacity to respond rapidly) would be shown to be the dominant influences on retarded-nonretarded differences in processing speed. This issue was addressed in Experiment 1. It is also interesting to consider whether extended training under conditions that constrain overly coarse trial-to-trial RT adjustments would lead to subsequent improvements in processing speed over and above the significant, but limited, effects that result from extended practice (see Brewer, 1987, for a review of practice studies). Experiment 2 focused on this second question, providing further information on the adjustability of processing speed.

\section{EXPERIMENT 1}

In Experiment 1, we compared the capacity of retarded and nonretarded individuals to process information rapidly and accurately by providing conditions that minimized the influence of retarded-nonretarded differences in the precision of trial-to-trial RT adjustments. In contrast to a selfpaced task in which the subject controls adjustments to processing time from trial to trial, processing time was controlled experimentally using a deadline procedure (cf. Pachella \& Fisher, 1969, 1972; Pachella, Fisher, \& Karsh, 1968). Under a deadline procedure, subjects are required to respond prior to the end of a specified time interval or deadline. By varying the deadline across trial blocks, subjects produce a range of fast responses, and the relation between processing speed and performance accuracy can be determined empirically. In this study, deadlines of 300,400 , and $600 \mathrm{msec}$ were used during serial four-choice RT performance to generate speedaccuracy, or RT-error, functions for mentally retarded young adults and nonretarded individuals of the same chronological age (following the position of Ellis, 1969).

\section{Method}

Subjects. The mentally retarded subjects were 16 volunteers $(8$ male, 8 female) from an adult training center. Their ages ranged from 18 to 29 years, with a mean of 22 . Wechsler Adult Intelligence Scale (WAIS) full-scale IQs ranged from 56 to 70 , with a mean of 62 . None of the mentally retarded subjects had clinical histories indicating organicity, physical disabilities that would affect responding, or medical conditions requiring drug treatment. The chronological age control group comprised 16 undergraduate volunteers ( 8 male, 8 female). Their ages ranged from 18 to 30 years, with a mean of 22; all were presumed to be of average or above-average IQ. All subjects had normal visual acuity.

Apparatus. Stimuli were four lens-topped neon bulbs, $15 \mathrm{~mm}$ in diameter, arranged at 22-mm centers in a horizontal row and flush with a mat black surround. A vertical yellow line, about $1 \mathrm{~mm}$ wide, divided this background in half so that the lights appeared to be separated into two groups of two. The stimulus display was at eye level, about $0.8 \mathrm{~m}$ from the subject. The subjects responded with four keys that were arranged to fit comfortably under the middle and index fingers of each hand. The response keys were Plexiglas cylinders, $13 \mathrm{~mm}$ in diameter, curved on top to fit fingertips, and projecting up $5 \mathrm{~mm}$ through a baseboard. When pushed with a pressure of about $140 \mathrm{~g}$, the keys traveled through an amplitude of about $2 \mathrm{~mm}$ and returned to resting position when released by the subject. Stimulus presentation and response recording was controlled by a minicomputer (DEC-LSI/11).

Procedure. The task was serial four-choice RT. The stimulusresponse relation was compatible, with the four stimuli from left to right corresponding to responses with the left-hand middle, lefthand index, right-hand index, and right-hand middle fingers, respectively. Stimuli appeared one at a time and remained on until a key was pressed. RT (to the nearest millisecond) was measured from stimulus onset to response-key depression, with the interval between response-key release and onset of the next stimulus being $1.5 \mathrm{sec}$. This interval is sufficiently long for any processing of the previous stimulus and response to be finalized by retarded subjects (Baumeister \& Kellas, 1968).

The RT task was performed under deadline intervals of 300,400 , and $600 \mathrm{msec}$. The expiration of the deadline interval was signaled 
by a loud buzzer emitted by the computer. The buzzer sounded only if the subject failed to respond before the deadline and continued to sound until a key was pressed. The subjects were instructed to respond prior to the onset of the deadline signal and, in so doing, to respond as accurately as possible.

In Session 1, each subject received training and 100 practice trials on the four-choice task (without deadlines). In Sessions 2, 3, and 4 , the subjects performed the task under deadlines of 600,300 , and $400 \mathrm{msec}$, respectively. Each of these sessions included 30 coaching trials at the appropriate deadline, 100 practice trials, and 200 experimental trials. In Sessions 5, 6, and 7, the deadlines were $\mathbf{4 0 0}$, 300 , and $600 \mathrm{msec}$, respectively. Each of these sessions comprised 30 warm-up trials and 200 experimental trials. In total, each subject completed 400 experimental trials at each of the three deadlines. In each 200-trial session, the four stimuli appeared with equal frequency within each block of $\mathbf{4 0}$ trials; otherwise, stimulus order was random. The sessions were spaced over 7 consecutive working days.

\section{Results and Discussion}

Table 1 shows mean RTs, error percentages, and the percentage of responses that beat the deadline for each deadline condition. Although the retarded subjects were less successful in beating the deadline, RTs approximating the respective deadlines indicate that the procedure was effective in controlling processing time.

To examine the relation between processing speed and accuracy, conditional error functions (CEFs) were obtained for each group using a technique derived from the Vincentizing procedure (Ratcliff, 1979). For each subject, RTs for all responses that beat the respective deadlines were partitioned into fifth percentiles, and the RT and percentage of error associated with each fifth percentile was determined. Then, for each fifth percentile, mean RT and percentage of error were obtained for the retarded and nonretarded groups. The resulting CEFs are shown in Figure 1. For both groups, the CEFs indicate that (1) at very short RTs, error rates were highest, and (2) as RT increased, accuracy improved, with long RTs associated with high accuracy.

The retarded subjects did not, however, attain the degree of accuracy achieved by the nonretarded subjects at any RT level. This observation was supported by a regression analysis in which percentage error for the retarded subjects at each fifth percentile was plotted as a function of percentage error for the nonretarded subjects. The resulting curve was best described by a linear function (retarded \% $=13.5+2.3$ nonretarded $\%$ ); $r^{2}$ $=0.86, p<.0001)$. The intercept $(13.5)$ was significantly different from zero $[t(18)=8.16, p<.0001]$, confirming the group differences in accuracy that prevailed even at relatively long $R T$ s.

The deadline procedure used in Experiment 1 minimizes the influence of those control mechanisms mediating the trial-to-trial adjustments in speed and accuracy that have been shown (Brewer \& Smith, 1984) to be important factors contributing to retarded-nonretarded differences in processing speed. The obtained relation between speed and accuracy demonstrated that when conditions are such that retarded individuals' responding is consistently rapid and equivalent in speed to that of nonretarded individuals, performance accuracy is lower. This result represents the strongest evidence to date of the existence of structural, or nonadjustable, limitations on processing speed in retarded individuals.

\section{EXPERIMENT 2}

Although Experiment 1 demonstrated a significant, nonadjustable component associated with the processingspeed differences between retarded and nonretarded individuals, to what extent-and by what means-speed on self-paced rapid-processing tasks is adjustable in retarded individuals remains a relevant and little-explored issue. Several studies have reported improvements in processing speed following the use of incentives (Baumeister \& Ward, 1967; Hasazi \& Allen, 1973; Holden, 1966), and particularly following extended practice or training (Brewer \& Smith, 1984; Hoover, Wade, \& Newell, 1981; Nettelbeck, Evans, \& Kirby, 1982; Phillips \& Nettelbeck, 1984). Although, in some studies, performance improvements have been marked, substantial differences between retarded and nonretarded samples have remained (Brewer, 1987). As yet, however, no research has been conducted

Table 1

Mean Reaction Times, Percentages of Errors, and Percentages of Responses that Beat the Deadline in Experiment 1

\begin{tabular}{|c|c|c|c|c|c|}
\hline \multirow[b]{2}{*}{ Subject } & \multicolumn{2}{|c|}{ Mean RT } & \multicolumn{2}{|c|}{ Percentage of Errors } & \multirow{2}{*}{$\begin{array}{l}\text { Percentage of } \\
\text { Responses that } \\
\text { Beat Deadline }\end{array}$} \\
\hline & Correct & Error & Range & Median & \\
\hline \multicolumn{6}{|c|}{ 300-msec Deadline } \\
\hline Nonretarded & 304 & 289 & $1.2-24.5$ & 9.8 & 50.5 \\
\hline Retarded & 344 & 314 & $4.2-74.5$ & 35.3 & 38.4 \\
\hline \multicolumn{6}{|c|}{ 400-msec Deadline } \\
\hline Nonretarded & 379 & 351 & $0.0-18.8$ & 2.8 & 60.5 \\
\hline Retarded & 454 & 424 & $0.5-38.5$ & 8.1 & 34.2 \\
\hline \multicolumn{6}{|c|}{ 600-msec Deadline } \\
\hline Nonretarded & 584 & 465 & $0.0-3.0$ & 0.6 & 57.1 \\
\hline Retarded & 666 & 657 & $0.0-28.3$ & 4.4 & 35.4 \\
\hline
\end{tabular}

Note-Mean RTs are expressed in milliseconds. 


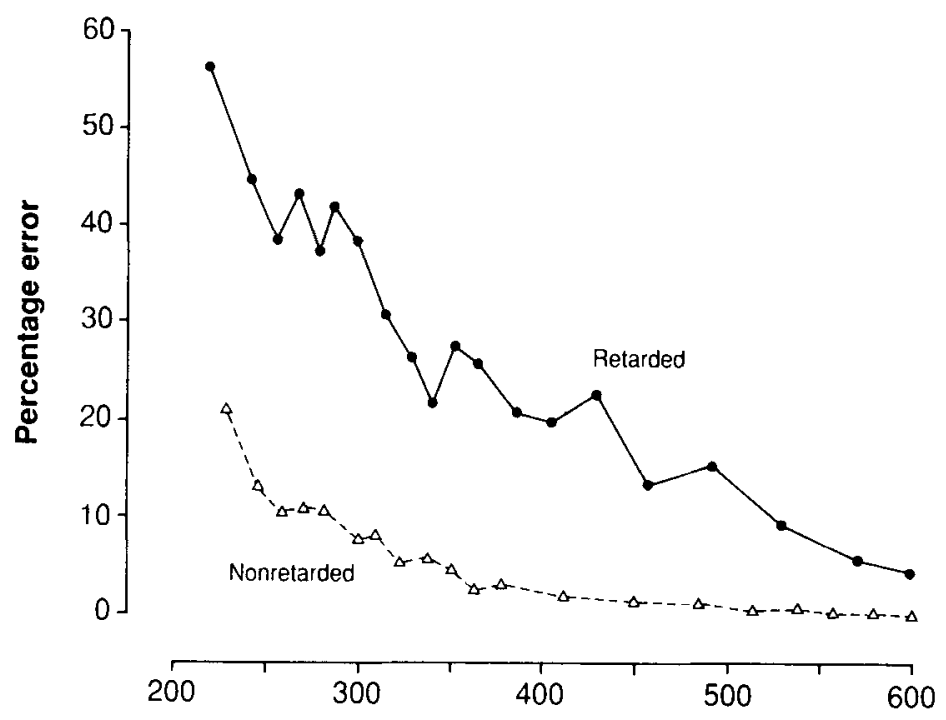

Reaction time in $\mathrm{ms}$

Figure 1. Experiment 1: Percentages of errors for retarded and nonretarded groups at each fifth percentile of the RT distributions for responses beating the deadlines.

in which attempts to adjust processing speed have focused directly on improving the efficiency of informationprocessing operations or mechanisms that have been shown to constrain performance efficiency in retarded individuals.

Experiment 2 addressed this issue by examining the effects of extended training aimed at controlling the overly coarse trial-to-trial RT adjustments that contribute to the slower, more variable responding of retarded individuals on self-paced, rapid-processing tasks (Brewer \& Smith, 1984). Retarded subjects' performance on a self-paced, serial choice RT task was examined prior to and following extended training at a short, fixed response deadline. The purpose was to establish whether retarded subjects could improve processing speed by learning to control RT adjustments within relatively narrow RT limits.

To determine whether improvements in processing speed following training exceeded those that would occur simply as a result of practice on the self-paced RT task, a multiple-baseline across-subjects design (Barlow \& Hersen, 1984) was employed. In this design, data for each subject are collected on a different baseline, with the introduction of the experimental intervention being staggered across baselines. The design, which has been widely used in clinical research, allows performance levels to be compared within groups. If, for example, performance changes in some consistent manner in all subjects following the intervention, regardless of where on the baseline the intervention occurred, this points to the influence of that intervention. In a multiple-baseline design, performance changes are typically monitored from the point at which the intervention is introduced. Experi- ment 2 was a variation on that procedure, with performance monitored from the completion of the intervention.

In Experiment 2, deadline training was introduced at different stages of practice for each subject. For 3 different subjects, deadline training was introduced after 1,000 trials, 2,000 trials, and 4,000 trials, respectively. All subjects then performed 5,000 trials under a fixed deadline of 350 msec. (CEFs obtained by RT partitioning following self-paced performance on an identical RT task [see Brewer \& Smith, 1984] indicated that retarded subjects could respond at an RT of $350 \mathrm{msec}$ with relatively low error rates of about $10 \%$. However, they did not achieve that RT level consistently-approximately $90 \%$ of RTs exceeded $350 \mathrm{msec}$.) After deadline training, the subjects completed $4,000,3,000$, or 1,000 trials, respectively, on the self-paced RT task, so that each subject performed a total of 5,000 trials on this task.

\section{Method}

Subjects. The subjects were 3 female volunteers from an adult training center for mentally retarded individuals. Their ages were 20,20 , and 23 years. Intelligence test scores were not made available, but the subjects were selected by the center's staff from their mildly retarded employees (WAIS Full-Scale IQs ranging from 55 to 69). Other selection criteria were the same as in Experiment 1. The subjects traveled independently to the laboratory and were paid for participating at the completion of the experiment.

Apparatus and Procedure. The apparatus and general task setup were the same as in Experiment 1.

All subjects received training and 200 practice trials on the fourchoice RT task (without deadline). They then were exposed, in order, to the following three conditions: four-choice RT without deadline, four-choice RT with a fixed deadline of $350 \mathrm{msec}$, and four-choice RT without deadline. A multiple-baseline across-subjects 
Table 2

Mean Reaction Times, Percentages of Errors, and Percentages of Responses that Beat the Deadline for Individual Subjects in Experiment 2

\begin{tabular}{|c|c|c|c|c|}
\hline \multirow{2}{*}{$\begin{array}{l}\text { Trial } \\
\text { Block }\end{array}$} & \multicolumn{2}{|c|}{ Mean RT } & \multirow{2}{*}{$\begin{array}{l}\text { Percentage } \\
\text { of Errors }\end{array}$} & \multirow{2}{*}{$\begin{array}{l}\text { Percentage of } \\
\text { Responses that } \\
\text { Beat Deadline }\end{array}$} \\
\hline & Correct & Error & & \\
\hline \multicolumn{5}{|c|}{ Subject A } \\
\hline $1-13$ & 342 & 336 & 10.9 & 84.8 \\
\hline $14-25$ & 325 & 313 & 12.1 & 90.1 \\
\hline Overall & 334 & 325 & 11.5 & 87.4 \\
\hline \multicolumn{5}{|c|}{ Subject B } \\
\hline $1-13$ & 350 & 331 & 47.5 & 84.9 \\
\hline $14-25$ & 351 & 336 & 43.4 & 85.4 \\
\hline Overall & 350 & 333 & 45.6 & 85.1 \\
\hline \multicolumn{5}{|c|}{ Subject C } \\
\hline $1-13$ & 595 & 528 & 22.6 & 29.0 \\
\hline $14-25$ & 413 & 418 & 40.3 & 60.9 \\
\hline Overall & 482 & 475 & 31.1 & 44.3 \\
\hline
\end{tabular}

Note-Mean RTs are expressed in milliseconds.

design was used, with the fixed deadline being introduced at a different stage of practice for each subject. The deadline was introduced after 1,000 trials for Subject A, after 2,000 trials for Subject B, and after 4,000 trials for Subject $C$. All subjects performed 5,000 trials under the fixed deadline, and then reverted to the nondeadline task to complete a total of 5,000 trials on that task. Thus, the 3 subjects performed 4,000,3,000, and 1,000 trials, respectively, after the deadline.

In the four-choice task without deadline, the subjects were instructed to respond as quickly as possible without making too many errors. The instructions in the deadline condition were the same as in Experiment 1. Trials were presented in blocks of 200 in both deadline and nondeadline conditions. The subjects attended 5 days a week, completing 1-3 blocks per day

\section{Results and Discussion}

Table 2 shows mean correct and error RTs, percentage error, and the percentage of responses that beat the deadline for Deadline Training Blocks 1-13, 14-25, and $1-25$. Although accuracy varied across subjects during the deadline condition, the deadline training was effective from the outset in controlling processing time for Subjects $A$ and $B$, with stable performance established after one or two trial blocks. For Subject C, stable performance in the vicinity of the deadline was not established until after 8-10 trial blocks. However, this still provided Subject $C$ with about 3,000 training trials at the targeted processing speed.

It is notable that, even after 5,000 trials at the $350-\mathrm{msec}$ deadline, the subjects did not show any noticeable improvements in accuracy. Also, they were unable to achieve accuracy levels comparable to those achieved by the nonretarded subjects under similar conditions. The error rates for the nonretarded subjects at the $300-$ and $400-\mathrm{msec}$ deadlines in Experiment 1 would predict an error rate somewhere between $4.2 \%$ and $10.4 \%$ at a $350-$ msec deadline, considerably lower than those produced by the retarded subjects (see Table 2). These two findings strengthen the conclusion of Experiment 1 regarding structural limitations on processing speed in retarded individuals.

To examine the effects of training under the fixeddeadline condition, changes in mean correct RT across trial blocks before and after training were plotted (see Figure 2). For each subject, mean RT was substantially shorter immediately following deadline training than it was prior to its introduction. Mean RTs for the first 1,000 trials beyond the deadline training were shorter than those for the 1,000 trials immediately before the deadline training by $255 \mathrm{msec}$ ( $43 \%$ reduction), $124 \mathrm{msec}$ ( $22 \%$ reduction), and $248 \mathrm{msec}$ ( $35 \%$ reduction) for Subjects A, B, and $C$, respectively. The $R T$ reductions from the initial trial block to the first postdeadline block were $336 \mathrm{msec}$ (50\%), $328 \mathrm{msec}(43 \%)$, and $701 \mathrm{msec}(58 \%)$, respectively.

These RT reductions occurred regardless of whether the deadline training was introduced relatively early or late in practice. While it might possibly be argued that the RT improvements resulted from the extra practice

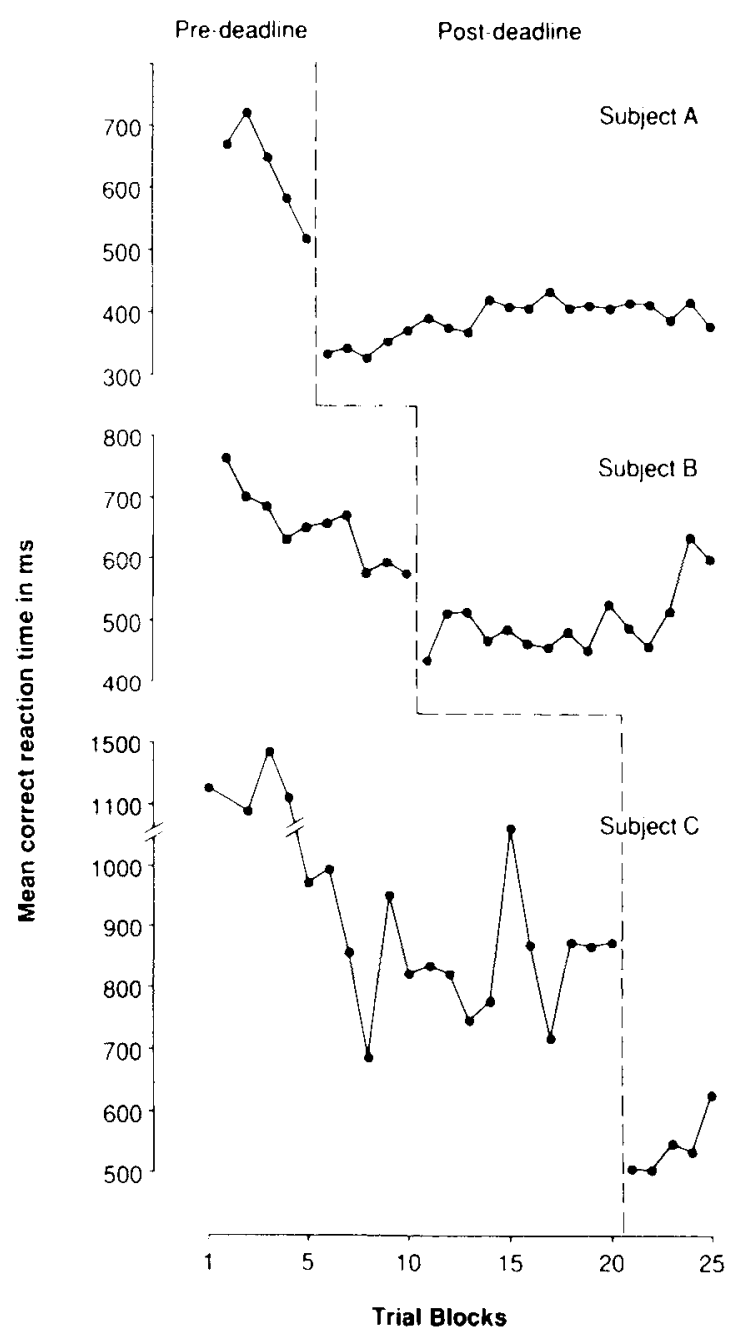

Figure 2. Experiment 2: Mean correct RTs for individual retarded subjects pre- and postdeadline training. 
Table 3

Percentages of Errors in Each Five-Trial Blocks Pre- and Postdeadline Training for Individual Subjects in Experiment 2

\begin{tabular}{|c|c|c|c|c|c|}
\hline \multirow[b]{2}{*}{ Subject } & \multicolumn{4}{|c|}{ Trial Block } & \multirow[b]{2}{*}{ Overal } \\
\hline & $1-5$ & $6-10$ & $11-15$ & $16-20$ & \\
\hline \multicolumn{6}{|c|}{ Predeadline } \\
\hline A & 2.9 & & & & 2.9 \\
\hline B & 6.8 & 8.9 & & & 7.8 \\
\hline $\mathrm{C}$ & 8.0 & 11.5 & 8.1 & 7.2 & 8.7 \\
\hline \multicolumn{6}{|c|}{ Postdeadline } \\
\hline A & 2.8 & 2.7 & 2.1 & 1.9 & 2.4 \\
\hline B & 5.0 & 9.8 & 9.1 & & 8.3 \\
\hline $\mathrm{C}$ & 8.7 & & & & 8.7 \\
\hline
\end{tabular}

gained in four-choice responding during the deadline training, several factors allow us to rule out this interpretation. First, Subject C showed substantial postdeadline RT reduction, despite having reached asymptote about 10 blocks prior to the introduction of deadline training. Second, a number of practice studies have indicated that, on various tasks (including one identical to that used here), retarded subjects reach asymptote within 2,000 trials at most (Brewer \& Smith, 1984; Hoover et al., 1981; Phillips \& Nettelbeck, 1984). This suggests that Subject B had probably also reached asymptote prior to the deadline training. And finally, the upward drift in mean RT and variability on the later postdeadline sessions (see later discussion) is inconsistent with an interpretation in terms of practice effects.

The observed RT reductions also could not be attributed to the subjects' responding less accurately following the deadline training. A comparison of error rates on the 1,000 trials (i.e., 5 trial blocks) immediately before and after the deadline (see Table 3 ) indicates improved postdeadline accuracy for Subjects A and B. For Subject C, a small deterioration in accuracy was evident, but the RT reduction was substantial.

Postdeadline RTs showed a gradual drift over trials back in the direction of predeadline RT levels. For Subject A, this was associated with a gradual reduction in error rate; for Subject B, the error rate increased (see Table 3). The substantially longer RTs on the last one or two blocks for Subjects $B$ and $C$ were accompanied by substantially lower error rates $(2.3 \%$ and $4.0 \%$, respectively). We suspect that this occurred because the subjects-knowing that it was the last session-were emphasizing accuracy in an effort to impress the experimenter.

Two further lines of evidence showed that the marked RT improvements following deadline training in fact resulted from the retarded subjects' learning to control RT adjustments within much tighter RT limits. First, examination of the error RT distributions for each subject for the 1,000 trials immediately preceding and following the deadline training (see Figure 3) revealed that deadline training not only affected correct RT, but also had a marked effect on error RTs. The predeadline respond- ing contained a substantial proportion of errors at relatively long RTs, a pattern previously reported by Brewer and Smith (1984) and indicative of transient fluctuations in attention and/or discriminability. Such errors were, however, much less prevalent following deadline training.

Our earlier research (Brewer \& Smith, 1984) indicated that the reduction of slow errors, by itself, will have a more general or widespread impact on overall RT variability. We showed, for example, that the retarded subjects' trial-to-trial adjustments in processing speed were primarily influenced by characteristics of the most recent preceding trials, with occasional atypically slow errors producing severe upward RT adjustments. These adjustments triggered long sequences of responding characterized by RTs well above the fastest safe RT levels, and also mitigated against the subjects' sustaining for a series of trials the very fast RT levels that they sometimes achieved. Thus, our previous work would suggest that the deadline training, by reducing those occasional fluctuations or lapses that culminate in slow errors, produces
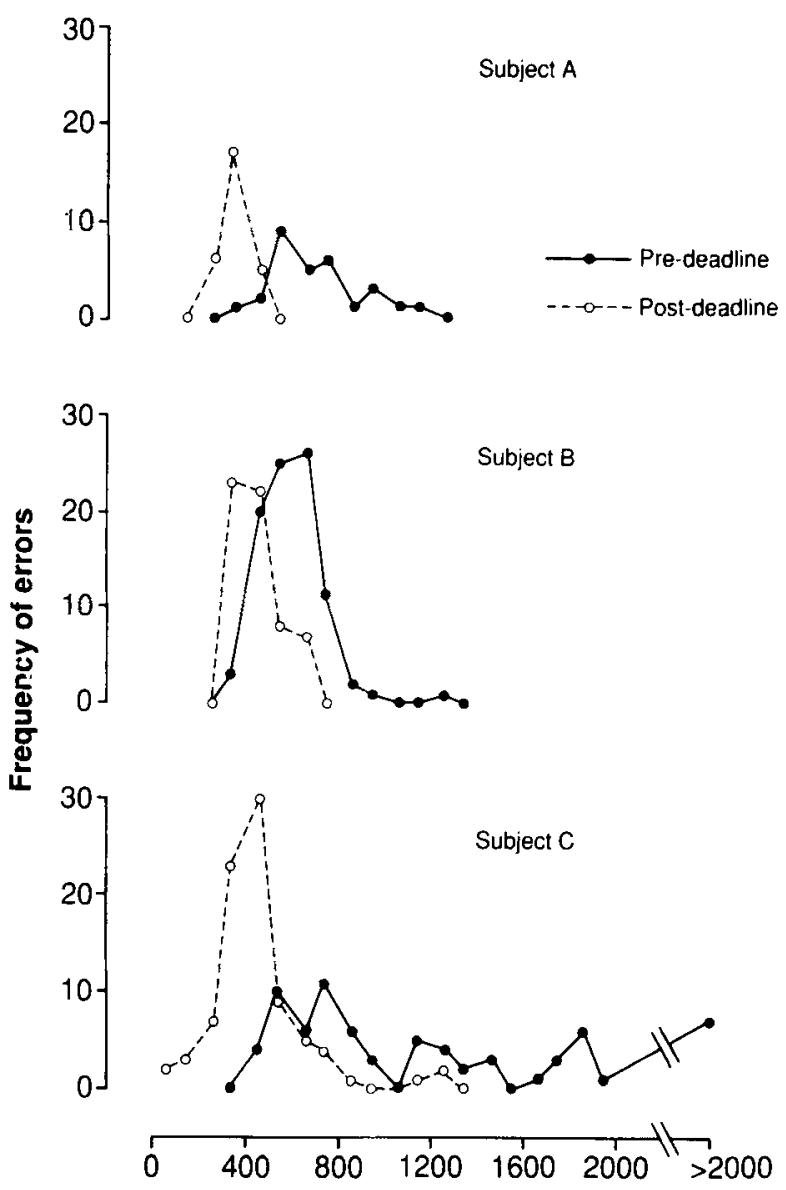

Reaction time interval in $\mathrm{ms}$

Figure 3. Experiment 2: Frequency of errors for individual retarded subjects pre- and postdeadline training for consecutive, discrete reaction time intervals of 100 msec. 


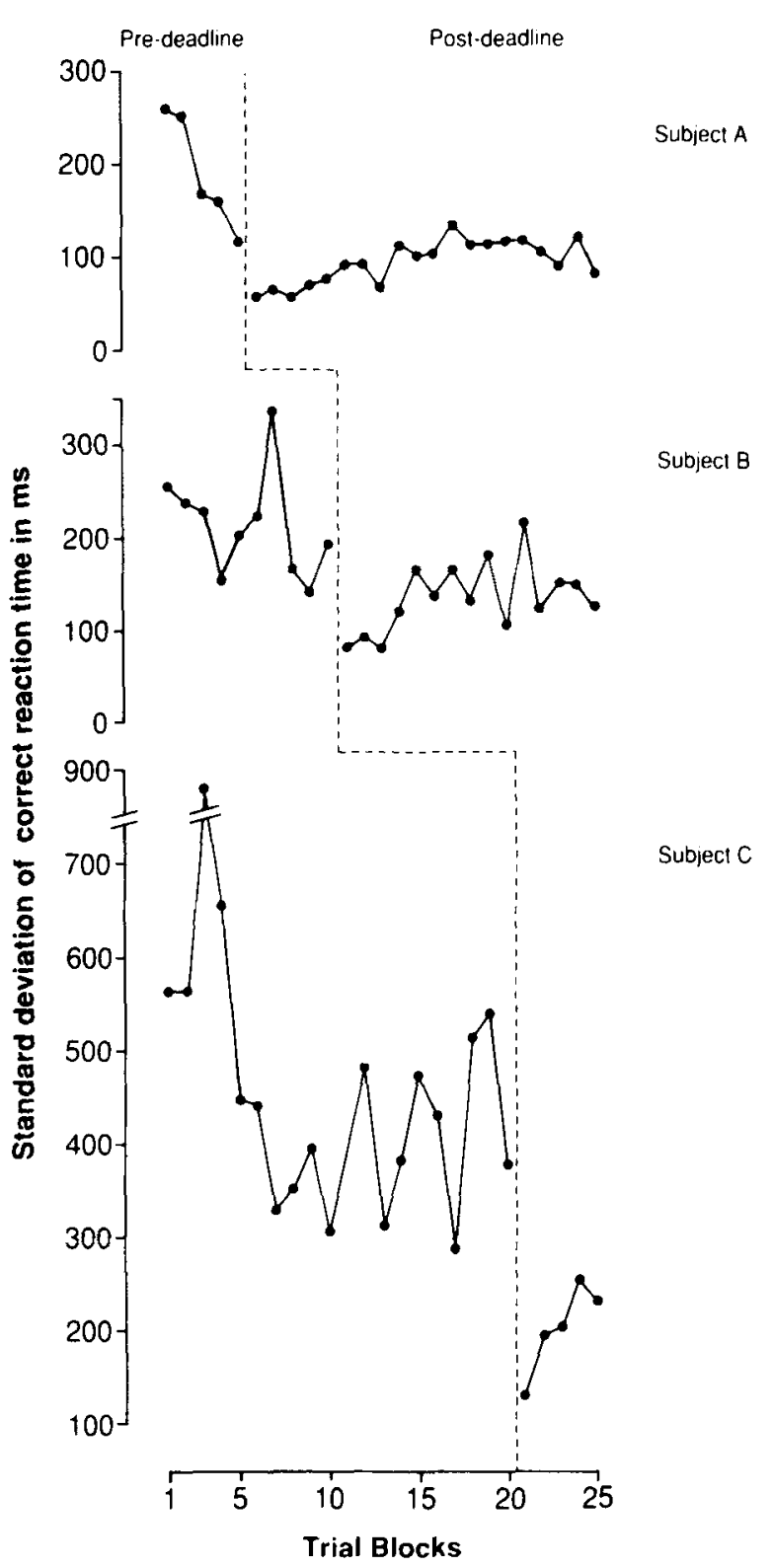

Figure 4. Experiment 2: Standard deviation of correct RTs for individual retarded subjects pre- and postdeadline training.

more controlled and adaptive (from the perspective of maximizing processing speed) RT adjustments. This, in turn, leads to reduced RT variability and average RT.

More direct evidence of the retarded subjects' tighter control of RT adjustments following the deadline training is provided in Figure 4, which shows the changes in standard deviation of correct RTs across trial blocks before and after the deadline training. For each subject, RT variability was substantially less immediately following deadline training than it was prior to its introduction. Over successive trial blocks, however, postdeadline RT variability for all subjects drifted back upward toward predeadline levels. The rate at which this occurred differed across subjects, with RT variability approaching predeadline levels within 1,000-2,000 trials.

\section{GENERAL DISCUSSION}

The two experiments reported here illustrate both fixed and adjustable limitations on processing speed of retarded individuals. In Experiment 1, we compared the capacity of retarded and nonretarded individuals to process information rapidly and accurately under conditions designed to minimize the influence of control mechanisms that previous research (Brewer \& Smith, 1984) has implicated in retarded-nonretarded differences in processing speed. A deadline procedure was used to equate retarded and nonretarded subjects' processing times. The resulting CEFs (speed-accuracy functions) demonstrated that the retarded subjects could not match the accuracy levels of the nonretarded subjects when responding as rapidly. This result represents compelling evidence for the existence of fixed or structural limitations on retarded individuals' processing speed. Further support for this conclusion came from Experiment 2, in which the retarded subjects, despite extended responding at a relatively short deadline, did not progress at all towards the accuracy levels achieved by the nonretarded subjects under similar deadline conditions.

The results of Experiment 2 also show, however, that substantial adjustments in retarded individuals' processing speed-beyond the effects of practice-can be achieved. Extended training at a fixed, short response deadline produced sharp reductions in RT (compared with baseline levels) when subjects were transferred back onto a self-paced task. The reductions occurred regardless of whether the deadline training was introduced early or late in practice. Error RT distributions and pre- and postdeadline RT variability suggest that the deadline training resulted in the retarded subjects' establishing much tighter control of RT adjustments than is typically exhibited in the absence of such training.

One issue that will need to be explored carefully in future studies is the durability of the effects of the deadline training. During the postdeadline stage, RT variability and, to a lesser extent, mean RT drifted back towards predeadline levels. Whether this pattern would become more marked with further trial blocks, and whether it could be controlled (e.g., by phasing out the deadline training gradually), are interesting questions for further research. A related question concerns the amount of deadline training needed to produce tighter control of RT adjustments.

A number of broader theoretical questions also arise from this research. Precisely what information-processing mechanism(s) mediates the improved control of RT adjustments shown by retarded subjects remains unanswered. Do, for example, the criterial adjustments underlying changes in mean RT and RT variability involve the direct regulation of processing time/speed or, alter- 
natively, the regulation of sensory evidence accumulated? Another question concerns the relationship between the findings reported here and other developmental data. Elsewhere (Brewer \& Smith, 1989), we have drawn attention to improved precision of RT adjustments as an important factor underlying developmental changes in processing speed in normal children. Further research with such populations along the lines pursued in the present study should help to refine our understanding of the similarities and differences in the information processing of retarded and nonretarded populations.

In summary, although this study confirmed the generally accepted position that the slower information processing of retarded individuals reflects structural limitations on processing efficiency, it also showed that this is only part of the story. Substantial adjustments to the processing speed of retarded individuals during serial choice RT performance were achieved by manipulations designed to improve the efficiency of control mechanisms associated with speed-accuracy regulation. Given that slower information processing impacts so broadly on the functioning of retarded individuals, this result provides some grounds for optimism. The challenge will be, however, to identify the control mechanisms constraining processing speed across a broad spectrum of tasks and to specify the conditions that will allow them to operate most efficiently.

\section{REFERENCES}

Barlow, D. H., \& Hersen, M. (1984). Single case experimental designs: Strategies for studying behavior change (2nd ed.). New York: Pergamon.

Baumeister, A. A., \& Kellas, G. (1968). Reaction time and mental retardation. In N. R. Ellis (Ed.), Intemational review of research in mental retardation (Vol. 3, pp. 163-193). New York: Academic Press.

BaUmeister, A. A., \& WARD, L. C., III (1967). Effects of rewards upon the reaction times of mental defectives. American Journal of Mental Deficiency, 71, 801-805.

BorkowsK, J. G., \& Cavanaugh, J. C. (1979). Maintenance and generalization of skills and strategies by the retarded. In N. R. Ellis (Ed.), Handbook of mental deficiency: Psychological theory and research (2nd ed., pp. 569-618). Hillsdale, NJ: Erlbaum.

Brewer, N. (1987). Processing speed, efficiency, and intelligence. In J. G. Borkowski \& J. D. Day (Eds.), Cognition in special children: Comparative approaches to retardation, leaming disabilities, and giftedness (pp. 15-48). Norwood, NJ: Ablex.

BREWER, N., \& SMITH, G. A. (1982). Cognitive processes for monitoring and regulating speed and accuracy of responding in mental retardation: A methodology. American Journal of Mental Deficiency, 87, 211-222.

BREWER, N., \& SMITh, G. A. (1984). How normal and retarded individuals monitor and regulate speed and accuracy of responding in serial choice tasks. Joumal of Experimental Psychology: General, 113, 71-93.

Brewer, N., \& SmITH, G. A. (1989). Developmental changes in processing speed: Influence of speed-accuracy regulation. Journal of $E x$ perimental Psychology: General, 118, 298-310.
Campione, J. C., \& Brown, A. L. (1977). Memory and metamemory development in educable retarded children. In R. V. Kail, Jr., \& J. W. Hagen (Eds.), Perspectives on the development of memory and cognition (pp. 367-406). Hillsdale, NJ: Erlbaum.

Detterman, D. K. (1979). Memory in the mentally retarded. In N. R. Ellis (Ed.), Handbook of mental deficiency: Psychological theory and research (2nd ed., pp. 727-760). Hillsdale, NJ: Erlbaum.

ELLIS, N. R. (1969). A behavioral research strategy in mental retardation: Defense and critique. American Journal of Mental Deficiency, $73,557-566$.

Fisher, M. A., \& Zeaman, D. (1973). An attention-retention theory of retardate discrimination learning. In N. R. Ellis (Ed.), International review of research in mental retardation (Vol. 6, pp. 169-256). New York: Academic Press.

HASAZI, J. E., \& ALLEN, R. M. (1973). Signal intensity and reinforcement effects on reaction time in brain-damaged and familial retardates. Perceptual \& Motor Skills, 36, 1227-1233.

HoldeN, E. A., JR. (1966). The effects of rest, reprimand, and reward on simple reaction time in educable retardates. American Journal of Mental Deficiency, 71, 427-432.

Hoover, J. H., WAde, M. G., \& Newell, K. M. (1981). Training moderately and severely mentally retarded adults to improve reaction and movement times. American Journal of Mental Deficiency, 85, 389-395.

Maisto, A. A., \& Baumeister, A. A. (1984). Dissection of component processes in rapid information processing tasks: Comparison of retarded and nonretarded people. In P. H. Brooks, R. Sperber, \& C. McCauley (Eds.), Learning and cognition in the mentally retarded (pp. 165-188). Hillsdale, NJ: Erlbaum.

NetTElBeCK, T., \& BREWER, N. (1981). Studies of mild mental retardation and timed performance. In N. R. Ellis (Ed.), International review of research in mental retardation (Vol. 10, pp. 61-106). New York: Academic Press.

Nettelbeck, T., Evans, G., \& Kirby, N. H. (1982). Effects of practice on inspection time for mildly mentally retarded and nonretarded adults. American Journal of Mental Deficiency, 87, 103-107.

Pachella, R. G. (1974). The interpretation of reaction time in information processing research. In B. H. Kantowitz (Ed.), Human information processing; Tutorials in performance and cognition (pp. 4182). Hillsdale, NJ: Erlbaum.

Pachella, R. G., \& Fisher, D. F. (1969). Effect of stimulus degradation and similarity on the tradeoff between speed and accuracy in absolute judgments. Joumal of Experimental Psychology, 81, 7-9.

Pachella, R. G., \& Fisher, D. F. (1972). Hick's law and the speedaccuracy tradeoff in absolute judgments. Journal of Experimental Psychology, 92, 378-384.

Pachella, R. G., Fisher, D. F., \& Karsh, R. (1968). Absolute judgments in speeded tasks: Quantification of the trade-off between speed and accuracy. Psychonomic Science, 12, 225-226.

Philuips, C. J., \& NetTelbeck, T. (1984). Effects of practice on recognition memory of mildly retarded adults. American Journal of Mental Deficiency, 88, 678-687.

RABBITT, P. (1979). How old and young subjects monitor and control responses for accuracy and speed. British Journal of Psychology, 70, 305-311.

RabBitT, P. M. A. (1981). Sequential reactions. In D. H. Holding (Ed.), Human skills (pp. 153-175). London: Wiley.

RATCLIFF, R. (1979). Group reaction time distributions and an analysis of distribution statistics. Psychological Bulletin, 86, 446-461.

(Manuscript received July 26, 1989; revision accepted for publication January 8,1990 .) 